\section{Long-term disorders of the blood-aqueous barrier after small- incision cataract surgery}

JÖRG SCHAUERSBERGER,

ANDREAS KRUGER,

ANDREA MÜLLNER-EIDENBÖCK,

VANESSA PETTERNEL,

CLAUDETTE ABELA, GERHARD SVOLBA, MICHAEL AMON

\section{Abstract}

Purpose To determine the long-term function of the blood-aqueous barrier after smallincision cataract surgery with implantation of a foldable intraocular lens.

Methods The blood-aqueous barrier function in 74 eyes of 62 patients who underwent cataract surgery was examined using a laser flare-cell meter. The measurements were performed pre-operatively and postoperatively between 12 and 35 months after surgery. For statistical analysis a linear regression was used. The study was designed as a single cohort study, with comparison of pre- and post-operative values.

Results Highly statistically significant differences $(p<0.0001)$ were found between pre-operative flare values and those measured at the final visit. The linear regression model showed significantly higher flare values postoperatively compared with those measured pre-operatively. Other variables such as incision technique, sex, operation time, phaco time and systemic disease had no influence on this outcome.

Conclusion The results suggest that there is persistent blood-aqueous barrier dysregulation even several years after cataract surgery.

Key words Aqueous humour, metabolism; Blood-aqueous barrier; Inflammation, aetiology; Lasers, diagnostic-use; Lens implantation, intraocular; Phacoemulsification, adverse effects

Many papers concerning the re-establishment of the blood-aqueous barrier (BAB) after cataract surgery have been published during the last decade. Using anterior chamber fluorophotometry, Sanders et al. ${ }^{1}$ observed reestablishment of the BAB within 3 months after extracapsular cataract surgery with intraocular lens implantation. Ferguson et al., ${ }^{2}$ also described recovery within the same time in about $80 \%$ of their patients; the rest $(\sim 20 \%)$ showed persistent $\mathrm{BAB}$ damage which was found to be correlated with an abnormal pupil shape or posterior synechiae. Several studies published at this time report persistent $\mathrm{BAB}$ dysfunction for more than 6 months after cataract surgery. Kaff et al. ${ }^{3}$ and Majima ${ }^{4}$ observed significant differences in the anterior chamber fluorescein concentration ratios between the operated and normal eye, or the fellow eye, up to 6 months post-operatively. Kaiya $^{5}$ and Kimata and co-authors ${ }^{6}$ found these differences even up to 4 years after the operation.

At the beginning of the 1990s scientists started to use the laser flare-cell meter to examine $\mathrm{BAB}$ disorders by measuring aqueous flare and cells. Shah et al. ${ }^{7}$ as well as Alio and co-authors ${ }^{8}$ examined cataract patients with otherwise normal eyes, and found no significant difference between pre-operative and postoperative ( 3 months) aqueous flare values. To our knowledge there are no recent publications concerning the long-term effects (up to 1 year and more) of small-incision cataract operation on the $\mathrm{BAB}$, using the laser flare-cell meter. The aim of this study was to examine the integrity of the $B A B$ 1-3 years after small-incision cataract surgery.

\section{Materials and methods}

Seventy-four eyes of 62 patients who underwent cataract surgery were examined pre-operatively and post-operatively at their final visit between 12 and 35 months after surgery. All eyes were free of ocular diseases other than cataract, and operated on by our standard cataract procedure (Table 1). None of these patients had any systemic or local anti-inflammatory medication for 1 month before operation.

\section{Standard cataract procedure}

All surgery was performed by one experienced surgeon after peribulbar anaesthesia with $2.5 \mathrm{ml}$ of lidocaine $2 \%$ plus $2.5 \mathrm{ml}$ of bupivacaine $0.5 \%$. The surgical procedure consisted of a clear cornea incision or sclerocorneal tunnel preparation, using a $3.5 \mathrm{~mm}$ steel blade, followed by continuous curvilinear capsulorhexis (CCC) performed under sodium

\section{J. Schauersberger \\ A. Kruger \\ A. Müllner-Eidenböck \\ V. Petternel \\ C. Abela \\ Department of \\ Ophthalmology \\ University Hospital of Vienna \\ Vienna, Austria \\ G. Svolba \\ Institute of Medical Statistics \\ University of Vienna \\ Vienna, Austria}

Dr Jörg Schauersberger Universitätsklinik für Augenheilkunde AKH, Währinger Gürtel 18-20 A-1090 Vienna, Austria

Tel: +43 1404007931

Fax: +431404007932

e-mail:

joerg.schauersberger@

akh-wien.ac.at

Proprietary or financial interest: None

This study was presented as a poster at the ARVO meeting in Fort Lauderdale, Florida, 1998

(Invest Ophthalmol Vis Sci 1998;39:51)

Received: 2 June 1999 Accepted in revised form: 25 August 1999 
Table 1. Patient characteristics

\begin{tabular}{ll}
\hline Age (mean) & 74.1 years \\
Age (range) & $46-89$ years \\
No. of women & 55 \\
No. of men & 19 \\
Sclerocorneal incision & 27 \\
Clear cornea incision & 47 \\
No. of patients with hypertension & 31 \\
No. of patients with diabetes mellitus & 10 \\
$\quad$ (without retinopathy) & 5 \\
No. of patients with asthma bronchiale & $42.2 \mathrm{~s}$ \\
Phaco time (mean) & $15 \mathrm{~min}$ \\
Operation time (mean) &
\end{tabular}

hyaluronate (Healon). After hydrodissection and hydrodelineation with fortified balanced salt solution (BSS Plus), phacoemulsification was performed using the bimanual 'divide and conquer' technique. Cortical remnants were removed by irrigation-aspiration. The capsular bag was refilled with sodium hyaluronate (Healon) and a foldable posterior chamber lenses was implanted into the bag. For implantation we used onepiece plate-haptic silicone lenses $(n=64)$, three-piece acrylic lenses $(n=7)$ and three-piece MMA-HEMA lenses $(n=3)$. The viscoelastic was then meticulously removed.

All patients in this group received the same postoperative therapy (topical diclofenac sodium and combined betamethasone-neomycin sulphate eye drops four times daily) for 1 month. No operative or postoperative complications occurred during the time of observation.

\section{Measurement of blood-aqueous barrier function}

Aqueous flare and cells were measured using the laser flare-cell meter (Kowa FC-1000, Kowa Europe, Dusseldorf, Germany). All patients had undilated pupils during measurement. Five sequential scans were averaged; the background scatter on each scan was less than $8 \%$. Calibration of the laser flare-cell meter was conducted periodically according to the manufacturer's instructions.

The study was designed as a single cohort study, with comparison of pre- and post-operative values. For statistical analysis of these differences in flare values a linear regression model was used. The time of postoperative measurement, centred at 2 years, was used as independent variable. For the analysis of other

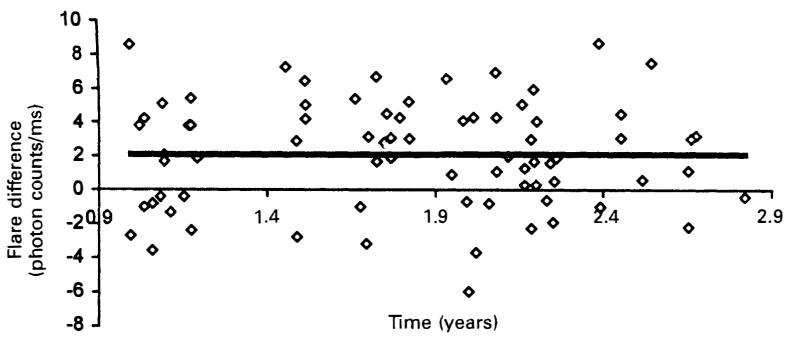

Fig. 1. Scatter-plot of the flare difference over time for the 74 eyes. The continuous line represents the linear regression. Each point represents the difference in aqueous flare between the pre-and post-operative measurement for one eye.
Table 2. Influence of other variables on the flare difference

\begin{tabular}{lc}
\hline Variable & $p$ value \\
\hline Eye (left/right) & 0.52 \\
Incision (CCI/SCI) & 0.69 \\
Sex (male/female) & 0.41 \\
Phaco time & 0.19 \\
Operation time & 0.22 \\
Diabetes mellitus & 0.72 \\
Hypertension & 0.42 \\
Anticoagulative drugs & 0.52 \\
Age & 0.0489 \\
\hline
\end{tabular}

independent variables a stepwise linear regression was performed. The variables needed a $p$ value lower than 0.05 to be included and to remain in the model.

\section{Results}

All 74 eyes had a mean pre-operative flare intensity of 7.62 photon counts $/ \mathrm{ms}(\mathrm{SD} \pm 2.70)$. The mean flare value of the final visit was 9.74 photon counts/ms ( $\mathrm{SD} \pm 3.29)$. The mean difference $(\Delta F)$ between pre- and postoperative values at 2 years postoperatively was 2.12 photon counts $/ \mathrm{ms}(p<0.0001)$. The time of the postoperative measurement had no influence on these results, because no difference was seen in $\Delta F$ determined between 1 and 3 years after surgery $(p=0.96)$ (Fig. 1). Of the variables eye (left/right), incision (SCI/CCI), sex, phaco time, operating time, diabetes mellitus, hypertension and use of anticoagulative drugs centred at 2 years, only age entered the stepwise regression model ( $p=0.0489$ ); however, this $p$ value explains only $6.75 \%$ of the variability. All other variables had $p$ values $>0.19$ (Table 2). There was no noticeable change when comparing the results including those patients with only the first operated eye with those results of both eyes.

\section{Discussion}

Any trauma in the eye, whether due to injury or surgery, results in a greater or lesser disturbance of the BAB. In the past, this functional disturbance was studied with special interest in patients after cataract surgery, as advances in surgical technique as well as materials and measuring procedures necessitate continuous, repeated evaluation of this phenomenon. While extracapsular cataract extraction with manual expression of the nucleus of the lens was considered a standard procedure in cataract surgery world-wide until recently, it has now been replaced by small-incision surgery with phacoemulsification. The latter is associated with much less trauma to the operated eye. ${ }^{9}$

At the same time, advances were made in postoperative therapy. The application of highly effective steroid- and non-steroid-based antiphlogistic drugs has markedly reduced post-operative inflammation and subsequent disturbance of the $\mathrm{BAB} .^{10,11}$ The same effect was achieved by the development of new lens material and design, which reduced the post-operative immunological defence reaction. ${ }^{12,13}$ Sophisticated 
measuring devices for measuring $\mathrm{BAB}$ disturbances (the current standard device being the laser flare-cell meter) permit a much more accurate evaluation of postoperative trauma than was achieved by previous methods (e.g. fluorophotometry). ${ }^{14}$ Moreover, as a result of these numerous developments, conflicting reports are found in the literature concerning whether and at which time point re-establishment of the BAB is achieved. This study highlights one aspect of this subject which has been given relatively less attention in the recent past, namely long-term observation of aqueous flare after cataract surgery. The results of our measurements showed significantly higher flare values 1-3 years after cataract surgery compared with those measured preoperatively.

Apart from the fact that these findings had no clinical significance, the results of our observation are surprising, as the majority of studies performed in a similar setting (healthy eyes, phacoemulsification, in-the-bag implantation) report re-establishment of the $\mathrm{BAB}$ within 3 months after surgery. ${ }^{7,8}$

This raises the question as to why persistent disturbance after cataract surgery is reported in spite of minimally invasive surgical techniques, modern medication and modern intraocular lens material. Analysis of our data led to the following conclusions: In our patients, systemic diseases and intra- or postoperative complications may be excluded as causes of persistent $\mathrm{BAB}$ disorders. One cause could be age-related changes in barrier function. Oshika et al. ${ }^{15}$ demonstrated a correlation between aqueous flare and age, which correlates with former observations ${ }^{16}$ of increased protein concentrations in older age groups. In contrast, our data show a markedly stronger increase in intracameral flare, especially in consideration of the relatively short time span of 1-3 years.

The assumption that this age-related barrier disorder is precipitated by surgery is also not true for the eyes we examined, In fact, we registered persistently increased flare values over 2 years of observation. Worthy of mention in this context is Kaiya's study, ${ }^{5}$ which reports constant $\mathrm{BAB}$ dysregulation over a period of 4 years (comparable with our observations). Some additional factors examined by us, such as operating time and phaco time, were also not correlated with the persistently increased flare values. Once these factors are excluded, it appears very likely that the sustained $B A B$ dysregulation we found is an expression of a persistent post-operative immunological reaction, probably a persistent foreign body reaction against the implanted lens. A few publications concerning this subject ${ }^{17-19}$ attribute a postoperative inflammatory reaction persisting 1 month postoperatively to this foreign body reaction. However, as yet this hypothesis is supported only by indirect data concerning post-operative cellular colonisation of the intraocular lens. The question concerning immunological processes and whether there are additional factors that favour this persistent $\mathrm{BAB}$ dysregulation must be investigated in further studies.

\section{References}

1. Sanders DR, Kraff MC, Liebermann HL, Peyman GA, Tarabishy S. Breakdown and reestablishment of blood-aqueous barrier with implant surgery. Arch Ophthalmol 1982;100:588-90.

2. Ferguson VMG, Spalton DJ. Continued breakdown of the blood aqueous barrier following cataract surgery. Br J Ophthalmol 1992;76:453-6.

3. Kaff MC, Sanders DR, Peyman GA, Lieberman HL, Tarabishy S. Slit-lamp fluorophotometry in intraocular lens patients. Ophthalmology 1980;87:877-80.

4. Mayima Y. Consideration of the modern cataract surgery. Acta Soc Ophthalmol Jpn 1982;100:588-90.

5. Kaiya T. Observation of the blood-aqueous barrier function after posterior chamber intraocular lens implantation. J Cataract Refract Surg 1990;16:320-4.

6. Kimata K, Nogawa H, Majima Y. Post-operative observation of the blood-aqueous barrier in pseudophakia (PLC) measured by fluorophotometry. Jpn Rev Clin Ophthalmol 1986;80:2190-3.

7. Shah SM, Spalton DJ. Changes in anterior chamber flare and cells following cataract surgery. Br J Ophthalmol 1994;78:91-4.

8. Alio JL, Sayans JA, Chipont E. Flare-cell meter measurement of inflammation after uneventful cataract surgery with intraocular lens implantation. J Cataract Refract Surg 1997;23:935-9.

9. Pande MV, Spalton DJ, Kerr-Muir MG, Marshall J. Postoperative inflammatory response to phacoemulsification and extracapsular cataract surgery: aqueous flare and cells. J Cataract Refract Surg 1996;22(Suppl 1):770-4.

10. Hessemer V, Schmidt KG, Schartner H. Minimally inflammatory cataract surgery. Klin Monatsbl Augenheilkd 1996;209:331-9.

11. Flach AJ, Dolan BJ, Donahue ME, Faktorovich EG, Gonzalez GA. Comparative effects of ketorolac $0.5 \%$ or diclofenac $0.1 \%$ ophthalmic solutions on inflammation after cataract surgery. Ophthalmology 1998;105:1775-9.

12. Miyake K, Ota I, Miyake S, Maekubo K. Correlation between intraocular lens hydrophilicity and anterior capsule opacification and aqueous flare. J Cataract Refract Surg 1996;22(Suppl 1):764-9.

13. Mester U, Strauss M, Grewing R. Biocompatibility and blood-aqueous barrier impairment in at-risk eyes with heparin-surface-modified lenses. J Cataract Refract Surg 1998;24:380-4

14. Spalton DJ, Shah SM. A comparative study of the laser flare cell meter and fluorophotometry in the assessment of the blood aqueous barrier in the pathological eye. Invest Ophthalmol Vis Sci (ARVO Suppl) 1991;32:886 (abstract 930).

15. Oshika T, Kato S, Sawa M, Masuda K. Aqueous flare intensity and age. Jpn J Ophthalmol 1989;33:237-42.

16. Brubaker RF, Nagataki S, Townsend DJ, Burns RR, Higgins RG, Wentworth W. The effect of age on aqueous humor formation in man. Ophthalmology 1981,88:283-8.

17. Miyake K, Asakura M, Kobayashi H. Effect of intraocular lens fixation on the blood-aqueous barrier. Am J Ophthalmol 1984;98:451-5.

18. Obstbaum SA. Biologic relationship between poly(methyl methacrylate) intraocular lens and uveal tissue. J Cataract Refract Surg 1992;18:219-31.

19. Pande M, Shah SM, Spalton DJ. Correlation between aqueous flare and cells and lens surface cytology in eyes with poly(methyl methacrylate) and heparin-surface-modified intraocular lenses. J Cataract Refract Surg 1995;21:326-30. 